\title{
Investigating stellar surface rotation using observations of starspots
}

\author{
Heidi Korhonen ${ }^{1,2,3}$ \\ ${ }^{1}$ Niels Bohr Institute, University of Copenhagen, Juliane Maries Vej 30, DK-2100 Copenhagen, \\ Denmark email: heidi.korhonen@nbi.ku.dk \\ ${ }^{2}$ Finnish Centre for Astronomy with ESO (FINCA), University of Turku, Väisäläntie 20, \\ FI-21500 Piikkiö, Finland \\ ${ }^{3}$ Centre for Star and Planet Formation, Natural History Museum of Denmark, University of \\ Copenhagen, Øster Voldgade 5-7, DK-1350, Copenhagen, Denmark
}

\begin{abstract}
Rapid rotation enhances the dynamo operating in stars, and thus also introduces significantly stronger magnetic activity than is seen in slower rotators. Many young cool stars still have the rapid, primordial rotation rates induced by the interstellar molecular cloud from which they were formed. Also older stars in close binary systems are often rapid rotators. These types of stars can show strong magnetic activity and large starspots. In the case of large starspots which cause observable changes in the brightness of the star, and even in the shapes of the spectral line profiles, one can get information on the rotation of the star. At times even information on the spot rotation at different stellar latitudes can be obtained, similarly to the solar surface differential rotation measurements using magnetic features as tracers. Here, I will review investigations of stellar rotation based on starspots. I will discuss what we can obtain from ground-based photometry and how that improves with the uninterrupted, high precision, observations from space. The emphasis will be on how starspots, and even stellar surface differential rotation, can be studied using high resolution spectra.
\end{abstract}

Keywords. stars: activity, stars: late-type, stars: rotation, stars: spots

\section{Introduction}

It is widely accepted that the global behaviour of the solar magnetic field can be explained by a dynamo action which is due to interaction between magnetic fields and fluid motions. The Sun is thought to have an $\alpha \Omega$-type dynamo, in which the poloidal field is created from the toroidal one by helical turbulence ( $\alpha$-effect), and the toroidal field is obtained by shearing the already existing poloidal field by differential rotation ( $\Omega$-effect). This kind of dynamo is also thought to work in other main-sequence stars with similar internal structure as the Sun has, i.e., stars with masses of $\sim 0.4-2.0 \mathrm{M}_{\odot}$.

Stellar rotation has a major impact on the over-all efficiency of the dynamo action, and thus on the level of observed magnetic activity. The relationship between rotation and activity was first studied in detail by Pallavicini et al. (1981) who investigated the correlation between the X-ray luminosity and projected rotation velocity, $v \sin i$. Since then several studies have shown that the magnetic activity increases with increasing rotation rate, until finding a rotation rate after which no increase, and even maybe a small decrease, occurs (e.g., Micela et al. 1985; Pizzolato et al. 2003; Wright et al. 2011). This so-called saturation limit of the magnetic activity is reached at certain spectral type dependent rotation period, which increases toward later spectral types (Pizzolato et al. 2003).

There is also evidence that the stellar cycle length correlates to some extend with the rotation rate. Faster rotation tends to create shorter activity cycles (e.g., Noyes 
et al. 1984; Saar \& Brandenburg 1999; Oláh et al. 2000). In a diagram where the cycle frequency $\left(\omega_{\text {cycl }}\right)$ is plotted against the rotational frequency $\Omega$ the stars also seem to occupy three different regions: so-called inactive, active, and super-active regions (e.g., Saar \& Baliunas 1992; Saar \& Brandenburg 1999).

The studies of open clusters with known ages show that young clusters, with age of some tens of Myrs, have many rapidly rotating cool stars. With increasing age the amount of cool rapidly rotating stars decreases, and around 600 Myrs the stars with similar internal structure to the Sun's have slowed down to relation that is dependent on stellar mass (see e.g., Barnes 2003; Meibom et al. 2011). The observed spin-down of the stars over their lifetimes can be attributed to the magnetic breaking, which is driven by mass-loss through a magnetised stellar wind (e.g., Skumanich 1972). Therefore, many young cool stars (spectral classes $\mathrm{G}-\mathrm{M}$ ) are rapid rotators. The rapid rotation introduces significantly stronger magnetic activity than is seen in their older main sequence counterparts, like the Sun. On the other hand, the Sun was most likely in its youth a very active stars. All the energetic events caused by the magnetic activity can heat the possible planet forming disc around the star and have an impact on its composition, thus also affecting the possible planet and planetary system formation process. The phenomena caused by stellar activity can also have similar effects on stellar brightness and radial velocity as orbiting planets, making it at times difficult to distinguish between planets and activity signatures (see, e.g., Queloz et al. 2001).

It is clear that stellar rotation has a significant effect on magnetic activity of stars. In this review I will discuss how to measure stellar rotation using starspots, and especially concentrating on detailed studies of stellar surface differential rotation, which is a crucial parameter in the solar and stellar dynamos.

\section{Methods for studying stellar rotation using starspots}

Stars are point sources and studying their surface features is very demanding. For a long time it was not possible to obtain direct, spatially resolved, images of the stellar surface, except in some very rare cases of near-by giant and supergiant stars, like Betelgeuse (Gilliland \& Dupree 1996). During the last years a breakthrough using long baseline infrared interferometers has occurred. Images with milli-arcsecond resolution can now be produced, and a variety of targets have been imaged with astonishing result, e.g., bulging stars rotating near their critical limit (Monnier et al. 2007), and images of the transiting disk in the $\epsilon$ Aurigae system (Kloppenborg et al. 2010). Infrared interferometric imaging has produced amazing results of stellar surfaces and the time is drawing near when even dark spots on cool stars can be imaged. In addition, helioseismology has given us an unprecedented view of the solar rotation, both at the surface and in the interior, but obtaining similar results for stars is still very much 'work in progress'.

The main methods for studying stellar rotation are similar to the ones used originally on the Sun, i.e., tracing the movement of magnetic features, mainly starspots. This can be done using photometry and high resolution spectroscopy through Doppler imaging techniques. The rest of the article is focused on these two methods. Still, it has to be remarked that stellar rotation has also been studied using spectral line profile shapes (e.g., Reiners \& Schmitt 2003) and Ca II H\&K emission variations (e.g., Donahue et al. 1996; Katsova et al. 2010).

\subsection{Photometry}

In many active stars the areas occupied by starspots are so large that they cause brightness variations which can be few tens of percent from the mean light level, thus easily 

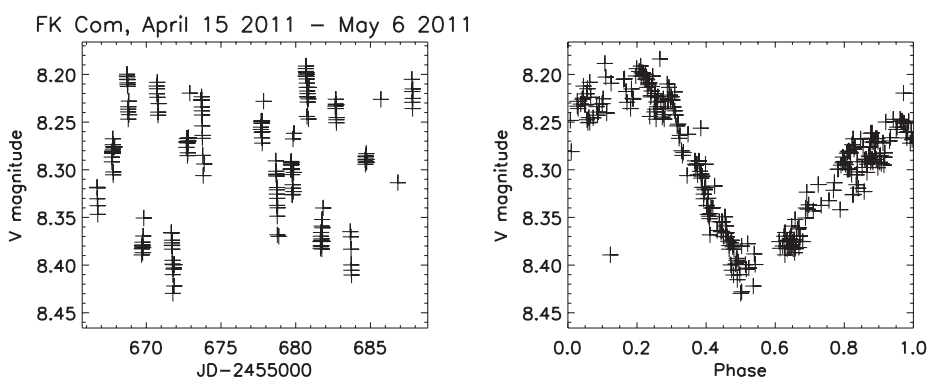

Figure 1. Example of ground-based observations of an active star FK Com. The plot gives the V magnitudes plotted against the Julian Date (left panel) and against the phase (right panel). The observations have been obtained in April-May 2011 using automatic photometric telescopes in Arizona. More information on the telescopes is given by Strassmeier et al. (1997).

observable even with small ground-based telescopes (see, e.g., Kron 1947). In comparison, biggest sunspots would only cause $\sim 0.01 \%$ decrease in the solar brightens and would require extremely precise instruments to detect.

Numerous studies of stellar rotation on active stars have been carried out based on photometry. Long-term monitoring campaigns have been carried out by several groups resulting in numerous papers on stellar rotation (e.g., Messina \& Guinan 2003; Strassmeier et al. 1997). Most of the campaigns are primarily used for studying long-term stellar cycles, but also the rotation periods and their possible variations have been investigated.

In general, the observations are carried out by automatic telescopes and typically a couple of observations per night are obtained during as many nights as possible within the observing season. Depending on the exact stellar rotation period this results in a time series from which the period might be difficult to determine accurately. Fig. 1 gives an example of a ground-based light-curve of an active star, in this case the single yellow giant FK Com. These observations actually present a good phase coverage light-curve obtained during a dedicated campaign. The typical dataset has much less observations within similar time period.

If the period is not straight forward to determine from the often sparse ground-based observations, it is one of the easiest stellar properties to obtain from continuous spacebased observations. With the recent launch of several photometric space missions, the high accuracy, high cadence, stellar light-curves are now also revolutionising the active star research. These missions include the Canadian micro-satellite MOST (for results on active stars see, e.g., Rucinski et al. 2004 and Siwak et al. 2011) and the French CoRoT satellite (e.g., Lanza et al. 2009; Silva-Valio et al. 2010; Huber et al. 2010). The real break-through happened, though, with the launch of the NASA's Kepler satellite. Kepler provides unprecedented accuracy light-curves of about 150000 stars, among them several active stars (see Fig. 2 and, e.g., Frasca et al. 2011). Based on GALEX data a sample of about 200 active stars have been selected for Kepler observations in a Guest Observer programme (Brown et al. 2011). The space-based data has shown us that basically every stellar rotation has slightly different spot configuration. This casts serious doubts in the old practice of using ground-based data from several rotations together.

\subsection{Doppler imaging}

Doppler imaging is a method that can be used for detailed mapping of the stellar surface structure (e.g., Vogt et al. 1987; Piskunov et al. 1990). Hereby high resolution, high signal-to-noise spectra at different rotational phases are used to measure the rotationally modulated distortions in the line-profiles. These distortions are produced by the 


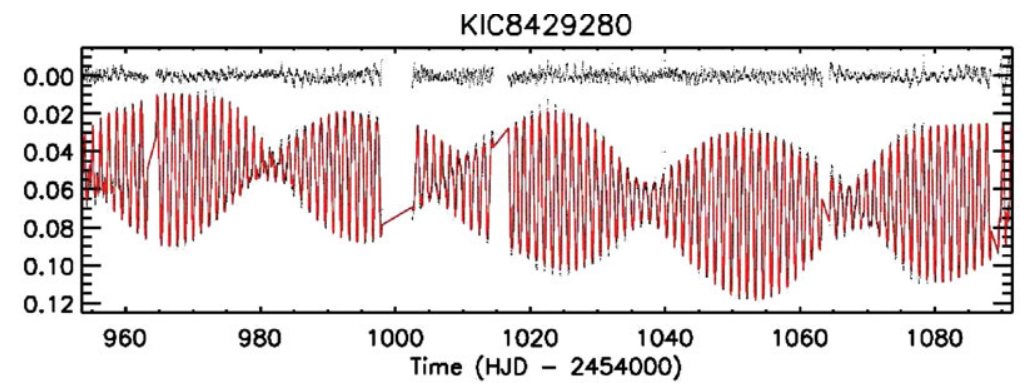

Figure 2. Example of a high precision continuous space-based observations of a young active star. The observations have been obtained using Kepler and have been published by Frasca et al. (2011).

inhomogeneous distribution of the observed characteristic, e.g., effective temperature, element abundance or magnetic field. Surface maps, or Doppler images, are constructed by combining all the observations from different phases and comparing them with synthetic model line profiles. Doppler imaging techniques were first used in the abundance mapping of Ap stars. Nowadays, Doppler imaging is more commonly used for temperature mapping of rapidly rotating late type stars (e.g., Korhonen et al. 2007; Skelly et al. 2010).

One has to keep in mind though, that for successful Doppler imaging a priori knowledge of the stellar rotation, usually based on earlier photometric investigations, is needed. If the rotation period of the Doppler imaging target is not know, it is very difficult to plan the observations so that a good phase coverage needed for Doppler imaging is obtained. Also, if the rotation period is not known accurately, comparison of the maps recovered at different epochs is not straight forward. The spectra and the variations seen in them can be used for estimating the rotation period of the target, but still the best results are obtained if also long-term photometric monitoring is carried out for accurate period determination.

Until autumn 2011 Doppler imaging has been applied to some 70 stars. Strassmeier (2009) gives a recent review on starspots and their properties. Here only a short summary on the main results we have learned about starspots using Doppler imaging is given.

Due to the enhanced magnetic activity in rapidly rotating cool stars, the spots are much larger than the spots observed in the Sun. The largest starspot recovered with Doppler imaging is on the active RS CVn binary HD 12545 which, in January 1998, had a spot that extended approximately $12 \times 20$ solar radii (Strassmeier 1999 ). The image of this starspot is shown in Fig. 3.

The lifetime of the large starspots can also be much longer than that of the sunspots, even years instead of weeks for sunspots (e.g., Rice \& Strassmeier 1996; Hussain 2002). One has to keep in mind though, that the spatial resolution obtained with Doppler imaging is not good enough for distinguishing whether the large spots are one single spot or a group of spots. If they are groups of spots, the individual spots could exhibit much shorter lifetimes than the group itself. Based on numerical simulations Işı et al. (2007) have shown that in a rapidly rotating active star the expected spot lifetime is few months, depending on the spot latitude (mid-latitude spots live a shorter time than equatorial or high latitude spots) and spot size (larger spots live longer). For sub-giants even longer spot lifetimes are obtained.

The latitudes at which starspots often occur are very different from those for the sunspots. In rapidly rotating late type stars spots can appear at very high latitudes, unlike in the Sun. This can be explained by the increase in the Coriolis force induced 


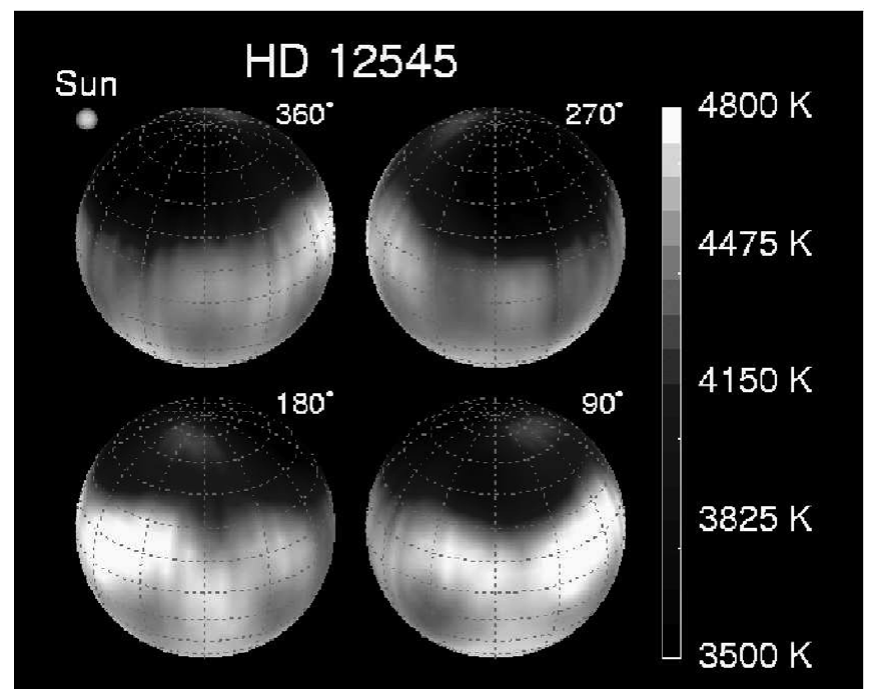

Figure 3. Doppler imaging results of HD 12545 by Strassmeier (1999).

by the rapid rotation (e.g., Schüssler \& Solanki 1992; Granzer et al. 2000). Still, these calculations cannot explain the formation of the polar caps, i.e., spots that are located at the rotational poles of the star, except in very young stars. These polar caps are still often seen in the Doppler images of also older late type stars (e.g., Weber \& Strassmeier 2001).

\section{Surface differential rotation}

Gaseous bodies, like the stars, can rotate differentially, i.e., different latitudes can have different rotation rates. In the Sun the rotation velocity of the photosphere depends strongly on the latitude; the rotation of the solar equator is approximately $30 \%$ shorter than the period at the poles. Helioseismological studies show that this latitude dependence persists throughout the convection zone (e.g., Thompson et al. 1996).

Differential rotation is one of the main elements in the dynamo models. Together with the helical turbulence and meridional flow it is responsible for the main features of the solar and stellar magnetic activity (see, e.g., Rüdiger et al. 1986; Brun \& Toomre 2002). Therefore, it is important to also measure differential rotation on other stars than the Sun.

Usually it is assumed that the differential rotation law of the Sun can be generalised to stars, leading the surface rotation law to be expressed by

$$
\Omega=\Omega_{\mathrm{eq}}+\beta \sin ^{2} \psi,
$$

where $\psi$ is the latitude, $\Omega_{\mathrm{eq}}$ is the equatorial angular velocity and $\beta$ defines the magnitude of the differential rotation. The relative differential rotation coefficient is given by

$$
\alpha=\frac{\Omega_{\mathrm{eq}}-\Omega_{\mathrm{pol}}}{\Omega_{\mathrm{eq}}} \text { or } \alpha=\frac{-\beta}{\Omega_{\mathrm{eq}}}
$$

where $\Omega_{\text {pol }}$ is the polar angular velocity. Note though that the exact formulation, symbols and signs, vary from author to author. Therefore, one has to be careful when comparing different works. 


\section{b. CCF map from Fer 6430}
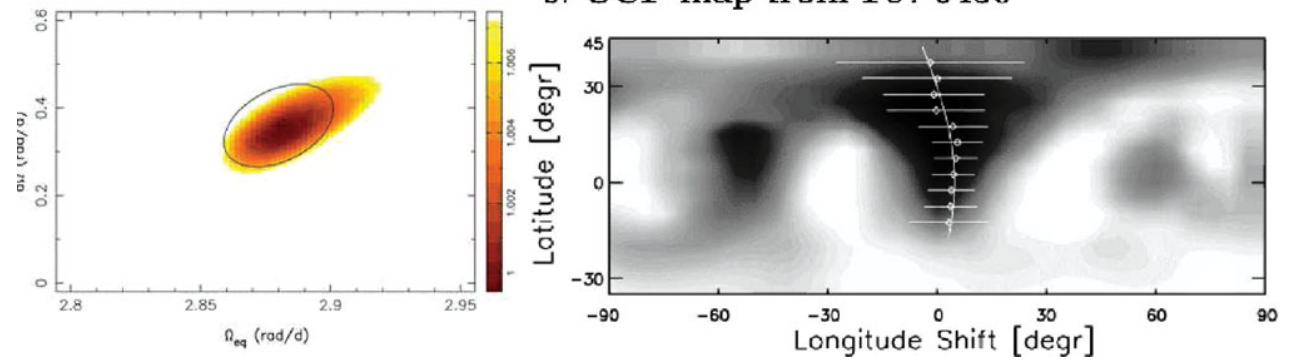

Figure 4. Examples of surface differential rotation determination using $\chi^{2}$-landscape (left; Marsden et al. 2011) and cross-correlation (right; Kővári et al. 2007a) methods.

Measuring stellar differential rotation is not straight forward. Currently the best way to study stellar surface differential rotation in detail, both the strength and the sign, is by using surface maps obtained with Doppler imaging. One way, similar to using sunspots and other magnetic features to study the solar surface differential rotation, is to crosscorrelate several surface maps obtained at different times with Doppler imaging. This allows to investigate the changes in the locations of the spots and how that depends on the latitude (see, e.g., Barnes et al. 2000; Weber et al. 2005; Kővári et al. 2007a). The other often applied method is so-called $\chi^{2}$-landscape technique in which a solarlike surface differential law is implemented into the Doppler imaging code, and many maps are obtained to study which parameters give the best solution (e.g., Petit et al. 2002; Marsden et al. 2006; Dunstone et al. 2008). For examples of both methods, look at Fig. 4. Stellar surface differential rotation has also been studied by combining spot latitude information from Doppler images and spot rotation period from simultaneous photometry (Korhonen et al. 2007).

\subsection{Surface differential rotation with spectral type and rotation period}

Donahue et al. (1996) investigated the rotation periods and the change in the rotation period, i.e., the magnitude of the surface differential rotation, in 36 stars from Ca II H\&K S-index measurements. They found that there was a power-law correspondence between these values, where $\Delta \Omega$ is proportional to the mean seasonal rotation, $\Omega$, with the power of 0.7, independent of mass. Barnes et al. (2005) collected measurements from different sources and methods, and obtained results which also imply power-law correlation between the rotation and surface differential rotation, but with a power of only 0.15 . On the other hand, recent investigation by Saar (2011) gives relation $\Delta \Omega \propto \Omega^{0.68}$, i.e. similar to the Donahue et al. (1996) results. It is clear that selecting homogeneous sample for these studies is difficult. Additionally, the surface differential rotation estimates used in some of these studies come from different methods and exhibit different systematic effects, therefore making comparison difficult.

The theoretical calculations by Kitchatinov \& Rüdiger (1999) show that the absolute value of the surface differential rotation decreases initially as the rotation period decreases from the solar value, but changes to a slight increase for periods of few days. They also predict that the differential rotation for the giant stars is larger than that for the dwarfs. Surface differential rotation measurements of 10 young G2-M2 dwarfs were obtained using Doppler imaging by Barnes et al. (2005). These measurements show an increase in the magnitude of differential rotation towards earlier spectral types, which is consistent with the theoretical calculations. Similar results have also been obtained by Saar (2011). 
Küker et al. (2011) carried out an theoretical study of rotation of G dwarfs. They computed model convection zones of different depth and investigated their large-scale gas motions, i.e. rotation and meridional flow, and compared the results to observations. Their calculations could easily produce the rotation laws of the slowly rotating Sun and several rapidly rotating $\mathrm{G}$ dwarfs. Their calculations failed to explain the extreme surface shear of HD 171488 (reported, e.g., by Marsden et al. 2005 and Jeffers \& Donati 2009), except when using an artificially shallow convection zone. This high-lights the fact that even though theory and observations at times encouragingly agree, there are still many unexplained features in the solar and stellar magnetic activity.

\subsection{Anti-solar differential rotation}

In general, models for global circulation in outer stellar convection zones predict solartype differential rotation, where the equator is rotating faster than the poles. However, Kitchatinov \& Rüdiger (2004) have shown that anti-solar differential rotation could arise as a result of intensive meridional circulation.

Anti-solar differential rotation, where the polar regions rotate faster than the equator, has been suggested by observations of several active stars (e.g., Vogt et al. 1999; Weber 2007; Kővári et al. 2007b). Kővári et al. (2007b) investigated surface flow patterns on $\sigma$ Gem from a series of observation spanning 3.6 consecutive rotation cycles, and found an anti-solar differential rotation with the surface shear of $-0.022 \pm 0.006$ (approximately $1 \%$ of the shear in the Sun, but of opposite sign). Additionally, they found evidence of a poleward migration trend of spots with an average velocity of $\sim 300 \mathrm{~m} / \mathrm{s}$. The strong meridional flow hinted at $\sigma$ Gem would support the hypothesis of Kitchatinov \& Rüdiger (2004), which attributes the anti-solar differential rotation to strong meridional circulation. Similar trend is seen by Weber (2007) in the investigation of several active stars. One has to note though, that these meridional flow measurements can arise from artefacts in maps and have to be confirmed with data from several epochs.

\subsection{Temporal variations in surface differential rotation}

Intriguingly, temporal evolution of the surface differential rotation has been reported for two young single K stars, AB Dor and LQ Hya (see, e.g., Donati et al. 2003; Jeffers et al. 2007). And in this case the question is not about small-scale variations, like seen on the Sun, but changes that are as large as $50 \%$ of the mean $\Delta \Omega$. Donati et al. (2003) hypothesise that these temporal variations could be caused by the stellar magnetic cycle converting periodically kinetic energy within the convective zone into large-scale magnetic fields and vice versa, as originally proposed by Applegate (1992). They also remark that a definite demonstration of the temporal variation would require monitoring a few stars for a long time, and seeing both the differential rotation parameters and the activity proxies showing the same cyclical variations, or at least to exhibit strongly correlated fluctuations in the case of non-cyclic behaviour.

\subsection{Cautionary remark on surface differential rotation studies}

A recent study by Korhonen \& Elstner (2011) used snapshots from dynamo models to investigate how well the cross-correlation method reproduces the latitudinal rotation rates used in the dynamo calculations. Their investigation showed that the cross-correlation method works well when the time difference between the maps is appropriate for recovering the surface differential rotation, and, importantly, if small-scale fields were included in the dynamo calculations. Using only the large-scale dynamo field the solution was dominated by the geometry of the dynamo field and the input rotation law was not recovered from the snapshots. Actually, the results in these cases showed much smaller 
surface differential rotation, than what was used in calculating the dynamo models. On the other hand, with additional injection of small-scale fields the input surface rotation law was well recovered.

This raises the question whether the large starspot seen in active stars can actually be created by small scale fields. If they are manifestations of the large-scale dynamo field, then according to the study by Korhonen \& Elstner (2011) we would not even expect them to follow the surface differential rotation. Their results also show that the exact latitude dependence of the rotation changes during the stellar cycle. Which could explain the temporal variation of surface differential rotation discussed above.

\section{Concluding remarks}

On the whole photometry is the easiest and least time consuming way of carrying out stellar rotation studies. Long time series of observations can easily be obtained for even relatively faint targets. Still, the usual cadence for this kind of observations is few observations per night - at most. This usually results in data from several different stellar rotation been used together, and also in difficulties to pin-point the stellar rotation period accurately. Continuous high cadence space-based observations have shown us that the light-curves of active stars change from rotation to rotation. Thus the ground-based observations usually give us only an average spot configuration. The high precision lightcurves from space missions, like CoRoT and Kepler, on the other hand provide us a unique opportunity to investigate stellar rotation and starspots with high temporal resolution. Actually, measuring the stellar rotation period from ground-based observations is often challenging, whereas from CoRoT and Kepler light-curves that is one of the easiest properties to determine.

The strength of the ground-based observations lies in investigating stellar cycles. Such long-term time series are virtually impossible to obtain from space due to the limited mission lifetimes. Also, instrumental artefacts, like trends, are often a problem in spacebased instruments, again hampering accurate studies of stellar cycles. Whereas obtaining rotational periods with high precision can uniquely be done using space-based continuous photometry.

Surface differential rotation can be estimated using photometric observations. But the investigations suffer from the fact that the information on the spot latitudes is usually impossible to obtain, and thus no information on the sign of the differential rotation can be obtained. Also, the latitude range of the spots is unknown, therefore only a lower estimate of the magnitude of the differential rotation can be obtained. Although, from high precision space-based photometry it might be possible to obtain also the latitude information.

For obtaining the magnitude and sign of the surface differential rotation detailed surface maps are needed. Still, also the studies using Doppler images are not without problems. All the methods based on Doppler imaging suffer from the often restricted latitude range the starspots occur on, and from the possible artefacts in the maps. These artefacts can for example rise from incorrect modelling of the spectral line profiles. For example incorrect modelling of the line core would produce a signal which is always visible around zero velocity, i.e., would result in a polar spot. Additionally, in $\chi^{2}$-landscape technique a predefined solar-type rotation law is assumed and thus no other latitude dependence can be recovered. On the other hand, in cross-correlation the time difference between the maps is crucial, with too small difference no change has time to occur, and with too long difference spot evolution due to flux emergence and disappearance can occur. Therefore, 
it is very demanding to obtain reliable measurements of the stellar surface differential rotation.

Even though, these concluding remarks offer more concerns than definite answers, it does not mean that the future of studying stellar rotation is bleak. Doppler imaging keeps on offering us an intriguing picture of stellar surface features and the space-based photometry is opening a real golden era for stellar rotation studies. In the future also asteroseismology and infrared/optical interferometry have a role to play in the new discoveries.

\section{Acknowledgments}

The author acknowledges the support from the European Commission under the Marie Curie IEF Programme in FP7.

\section{References}

Applegate, J. H. 1992, ApJ 385, 621

Barnes, J. R., Collier Cameron, A., James, D. J., \& Donati, J.-F. 2000, MNRAS 314, 162

Barnes, J. R., Collier Cameron, A., Donati, J.-F., James, D. J., Marsden, S. C., \& Petit, P. 2005, MNRAS 357, L1

Barnes, S. A. 2003, ApJ 586, 464

Brown, A., Korhonen, H., Berdyugina, S. V., et al. 2011, in: D. P. Choudhary, \& K. G. Strassmeier (eds.), Physics of Sun and Star Spots, Proc. IAU Symposium No. 273 (Cambridge University Press), p. 78

Brun, A. S. \& Toomre, J. 2002, ApJ 570, 865

Donahue, R. A., Saar, S. H., \& Baliunas, S. L. 1996, ApJ 466, 384

Donati, J.-F., Collier Cameron, A., Semel, M., et al. 2003, MNRAS, 345, 1187

Dunstone, N. J., Hussain, G. A. J., Collier Cameron, A., et al. 2008, MNRAS, 387, 1525

Gilliland, R. L. \& Dupree A. K. 1996, ApJ (Letters) 463, L29

Granzer, Th., Schüssler, M., Caligari, P., \& Strassmeier, K. G. 2000, A\&\&A 355, 1087

Frasca, A., Fröhlich, H.-E., Bonanno, A., Catanzaro, G., Biazzo, K., \& Molenda-Zakowicz, J. 2011, A\& A 532, A81

Huber, K. F., Czesla, S., Wolter, U., \& Schmitt, J. H. M. M. 2010, A\&\&A, 514, A39

Hussain, G. A. J. 2002, AN, 323, 349

Işık, E., Schüssler, M., \& Solanki, S. K. 2007, AESA 464, 1049

Jeffers, S. V., Donati, J.-F., \& Collier Cameron, A. 2007, MNRAS 375, 567

Jeffers, S. V. \& Donati, J.-F. 2009, MNRAS 390, 635

Katsova, M. M., Livshits, M. A., Soon, W., Baliunas, S. L., \& Sokoloff, D. D. 2010 New Astron. 15,274

Kitchatinov, L. L. \& Rüdiger, G. 1999, A\&A 344, 911

Kitchatinov, L. L. \& Rüdiger, G. 2004, AN 325, 496

Kloppenborg, B., Stencel, R., Monnier, J. D., et al. 2010, Nature 464, 870

Korhonen, H., Berdyugina, S. V., Hackman, T., Ilyin, I. V., Strassmeier, K. G., \& Tuominen, I. 2007, $A \mathscr{E} A, 476,881$

Korhonen, H. \& Elstner, D. 2011, A\&\&A 532, A106

Kővári, Zs., Bartus, J., Strassmeier, K. G., et al. 2007a, A\&A 463, 1071

Kővári, Zs., Bartus, J., Strassmeier, K. G., et al. 2007b, A\&̊A 474, 165

Kron, G. E. 1947, PASP 59, 261

Küker, M., Rüdiger, G., \& Kitchatinov, L. L. 2011, A\&A 530, A48

Lanza, A. F., Pagano, I., Leto, G., et al. 2009, A $\dot{E A} 493,193$

Marsden, S. C., Waite, I. A., Carter, B. D., \& Donati, J.-F. 2005, MNRAS 359, 711

Marsden, S. C., Donati, J.-F., Semel, M., Petit, P., \& Carter, B. D. 2006, MNRAS 370, 468

Marsden, S. C., Jardine, M. M., Ramirez Vélez, J. C., et al., 2011, MNRAS, 4131939 
Meibom, S., Barnes, S. A., Latham, D. W., et al., 2011, ApJ (Letters) 733, L9

Messina, S., \& Guinan E. F. 2003, A\&SA 409, 1017

Micela, G., Sciortino, S., Serio, S., et al. 1985, ApJ 292, 172

Monnier, J. D., Zhao, M., Pedretti, E., et al. 2007, Science 317, 342

Noyes, R. W., Weiss, N. O., \& Vaughan, A. H. 1984, ApJ 287, 769

Oláh, K., Kolláth, Z., \& Strassmeier, K. G. 2000, A\& A 356, 643

Pallavicini, R., Golub, L., Rosner, R, et al. 1981, ApJ 248, 279

Petit, P., Donati, J.-F., \& Collier Cameron, A. 2002, MNRAS 334, 374

Piskunov, N. E., Tuominen, I., \& Vilhu, O. 1990, A\&SA 230, 363

Pizzolato, N., Maggio, A., Micela, G., Sciortino, S., \& Ventura, P. 2003, A\&ऽA 397, 147

Queloz, D., Henry, G. W., Sivan, J. P. et al. 2001 A\&A 379, 279

Reiners, A., \& Schmitt, J. H. M. M. 2003 A $\& A$ 398, 647

Rice, J. B. \& Strassmeier, K. G. 1996, A\&A 316, 164

Rüdiger, G., Krause, F., Tuominen, I., \& Virtanen, H. 1986, A\&SA 166, 306

Rucinski, S. M., Walker, G. A. H., Matthews, J. M., et al. 2004, PASP 116, 1093

Saar, S. H. \& Baliunas, S. L. 1992, in: K. L. Harvey (ed), The Solar Cycle, Proceedings of the NSO/Sac Peak 12th Summer Workshop (Fourth Solar Cycle Workshop) (ASP Conf. Series, Vol. 27), p. 150

Saar, S. H. \& Brandenburg, A. 1999, ApJ 524, 295

Saar, S. 2011, in: D. P. Choudhary, \& K. G. Strassmeier (eds.), Physics of Sun and Star Spots, Proc. IAU Symposium No. 273 (Cambridge University Press), p. 61

Silva-Valio, A., Lanza, A. F., Alonso, R., \& Barge, P. 2010, A\& A510, A25

Siwak, M., Rucinski, S. M., Matthews, J. M., et al. 2011, MNRAS 415, 1119

Skelly, M. B., Donati, J.-F., Bouvier, J., et al. 2010, MNRAS 403, 159

Skumanich, A. 1972, ApJ 171, 565

Schüssler M., Solanki, S. K. 1992, A\&\&A 264, L135

Strassmeier, K. G., Bartus, J., Cutispoto, G., \& Rodono, M. 1997, A\&AS 125, 11

Strassmeier, K. G. 1999, A\&B A 347, 225

Strassmeier, K. G. 2009, Astronomy \& Astrophysics Review 17, 251

Thompson, M. J., Toomre, J., Anderson, E. R., et al. 1996, Science 272, 1300

Vogt, S. S., Penrod, G., \& Donald, Hatzes, A. P. 1987, ApJ 321, 496

Vogt, S. S., Hatzes, A. P., Misch, A., \& Kürster, M. 1999, ApJS, 121, 546

Weber, M. \& Strassmeier, K. G. 2001, A\& $A$ 373, 974

Weber, M., Strassmeier, K. G., \& Washuettl, A. 2005, AN, 326, 287

Weber, M. 2007, AN 328, 1075

Wright, N. J., Drake, J. J., Mamajek, E. E., \& Henry, G. W. 2011, ApJ in press, arXiv1109.4634

\section{Discussion}

JefFrey Linsky: On the Sun we see spots and faculae or anti-spots, they rotate at the same period of the spots and they tend to limb brightening. Are you fitting the data but not representing well the real star by not considering anti-spots?

Heidi Korhonen: In Doppler imaging we also allow for hot regions in our maps. We also regularly see these regions and they are used in the differential rotation analysis together with the cool spots. It is true, though, that often in light-curve analysis hot spots are not included.

Katja Poppenhager: What do spot maps look like using the two methods Doppler and Keppler imaging?

HeIDi Korhonen: Unfortunately the Keppler targets are quite faint; so, it is difficult to get good enough observations for Doppler imaging. We have some candidates for this and are working on them. 
DiBYENDU NANDY: Is it possible to determine spot migration like in a butterfly diagram using Doppler imaging?

Heidi Korhonen: In principle it is possible. The problem is that we do not have Doppler images for many stars with the required time base. Besides the stars that have many maps spanning enough years unfortunately have mainly high latitude spots, which do not change latitude much. We would very much like to find butterfly diagrams on other stars.

MARK Giampapa: Do you have a general impression of spot properties as a function of spectral type? For example, for different convection zone depths, how would the spot properties change?

Heidi Korhonen: Our sample of Doppler imaged targets is still quite small and the activity too affected by many other parameters, especially rotation. It is difficult to find good targets for comparison, but in general young stars show faster variability than the older ones and the spot contrast is smaller for cooler stars than for hotter stars. 